\title{
Problème de la remontée des eaux salées par les écluses maritimes Exemple de l'écluse de Mardyck, à Dunkerque
}

\author{
The problem of seawater back-flow through harbour locks \\ Example of the Mardyck lock at Dunkirk
}

\author{
P. Monadier
}

Directeur Technique du Port Autonome de Dunkerque

\section{Introduction}

L'écluse de Mardyck est en service depuis la fin de 1967, soit depuis environ 13 ans.

N'ayant participé ni à l'étude de l'ouvrage, ni à sa réalisation, ni à sa mise en service, il m'est impossible de vous faire part d'une expérience personnelle sur sa conception.

Je ne puis que me borner à reprendre les indications des rapports et articles déjà publiés sur cette installation. Le présent exposé n'a donc aucun caractère original et fait notamment de très larges emprunts, tout particulièrement pour le paragraphe 6, au texte de la Conférence présentée en 1972 devant le Comité Technique de la S.H.F. par M.M. G. Giauffret, alors Directeur de l'Exploitation du Port Autonome de Dunkerque et B. Quetin, Ingénieur à Sogreah $\left({ }^{1}\right)$. J'examinerai successivement :

- les données du problème posé ;

- les solutions envisagées ;

- la description de l'écluse de Mardyck ;

- les essais sur modèle réduit.

- les constatations et mises au point effectuées à l'entrée en service de l'écluse.

\section{Les données du problème posé}

La région de Dunkerque est une région de polders couvrant une superficie d'environ 40000 ha, dont la plus grande partie se trouve à un niveau inférieur à celui de la mer. Cette région est quadrillée par un réseau hydraulique extrêmement dense, les "watergangs", qui ont pour objet de maintenir ces terres en état de culture et sont étroitement liés au réseau

(1) voir la Houille blanche, $n^{\circ} 2-3,1972$. de navigation intérieure qui, selon les endroits et les şaisons, fournit l'eau nécessaire à l'irrigation ou collecte les eaux de drainage. Les industries utilisent également l'eau douce des canaux.

Il est donc indispensable d'éviter la pollution de ces derniers par l'eau de mer qui pourrait provenir des bassins du port de Dunkerque lors du sassement des bâteaux aux écluses de raccordement de ces bassins et du réseau de navigation in térieure.

$\mathrm{Au}$ débouché du canal de Bourbourg, qui est à petit gabarit, dans les bassins à flot du port Est de Dunkerque, le problème a été résolu par la création d'un bief intermédiaire, qui :

- se raccorde au canal en eau douce par une écluse ordinaire, l'écluse du Jeu de Mail ;

- se raccorde aux bassins en eau salée du port par des écluses ordinaires et parallèles, les écluses des darses I et II ;

- est établi à un niveau plus bas à la fois que les bassins du port et que le canal;

- collecte l'eau salée provenant des sassements à partir ou à destination du port et l'eau douce provenant des sassements à partir ou à destination du canal ; - communique avec la mer à marée basse par un ouvrage éclusé pour évacuer l'eau saumâtre qu'il a recueillie.

Ce dispositif n'est pas satisfaisant :

- la pénétration du sel dans le canal n'est pas complètement évitée, car les sassements à l'écluse de raccordement au canal font pénétrer dans celui-ci une certaine quantité d'eau salée ;

- il est très coûteux de réalisation, car il implique la construction de deux écluses de hauteur de chute supérieure à la différence de niveau à racheter et d'ouvrages de raccordement du bief intermédiaire à la mer (canal exutoire muni d'un ouvrage éclusé) ; 
- il consomme beaucoup d'eau puisque toute l'eau douce servant à l'éclusage du côté du canal est perdue.

Les inconvénients de ce dispositif ont conduit à rechercher, lors de la réalisation $\mathrm{du}$ raccordement du canal à grand gabarit Dunkerque-Valenciennes aux bassins maritimes du port Est, une solution plus satisfaisante sur les plans de la pollution et de la consommation de l'eau douce et plus économique sur le plan de l'investissement, d'autant que les plans d'extension $\mathrm{du}$ port ne permettaient pas, en tout état de cause, d'envisager la réalisation d'un exutoire vers la mer.

\section{Les solu tions envisagées}

La solution du bief intermédiaire ayant été a priori éliminée pour les raisons indiquées ci-dessus, trois solutions ne comportant qu'une seule écluse ont été examinées dans l'étude préliminaire avant d'effectuer un choix définitif ayant fait l'objet d'un examen approfondi.

Elles comportaient toutes deux points communs : pompage de l'eau de mer pour effectuer la vidange et présence d'un bassin anti-salure du côté du canal.

- la vidange par gravité conduirait à l'envoi dans le canal à chaque éclusée d'une tranche d'eau salée correspondant à la dénivellation, soit environ $55 \mathrm{~T}$ de sel par cycle et par mètre de dénivellation. Il est donc apparu nécessaire d'au moins repomper cette tranche d'eau salée pour effectuer la vidange ;

- l'introduction de sel dans le canal apparaît inévitable quelle que soit la solution. On peut créer une barrière contre la propagation par gravité de ces eaux salées en disposant à la sortie de l'écluse une fosse profonde qui accumule ces eaux et permet ensuite de les éliminer par pompage.

Les trois solutions mises à l'étude furent les suivantes :

\section{a) Protection par rideau d'air}

Le remplissage de l'écluse se fait par gravité à partir du côté port et la vidange s'effectue par pompage. Pour réduire les échanges par courants de densité on place, à chaque extrémité de l'écluse, un rideau transversal de bulles d'air créé en injectant de 1'air comprimé dans une rampe transversale placée au fond du canal.

b) Aspiration de la langue salée et compensation des volumes déplacés par les bateaux

Il a été envisagé :

- d'arrêter le coin salé en le pompant à la sortie de l'écluse et en le réalimentant à l'autre extrémité de manière à créer un écoulement stationnaire ;

- de compenser par pompage le transfert des volumes déplacés par les bateaux, l'eau de pompage étant prise du côté adéquat.

C'est surtout du côté terre que l'opération de blocage du coin salé et de compensation des volumes est intéressante, car elle permet de conserver l'eau salée dans le sas.

\section{c) Changement total de l'eau}

La nécessité de prévoir des pompes importantes dans la solution précédente a conduit à examiner une autre solution, consistant à changer complètement la qualité de l'eau à chaque éclusée. Les opérations de vidange et de remplissage sont conduites de manière à obtenir un sas plein d'eau douce quand on l'ouvre du côté du canal et plein d'eau de mer quand on l'ouvre du côté du bassin. On s'affranchit ainsi des sujétions dues aux mouvements des bateaux. L'eau douce est bien entendu récupérée à chaque fois.

Il est rapidement apparu que la solution (a) n'était pas satisfaisante, car elle entraînait des pertes importantes d'eau douce et exigeait le repompage d'une grande quantité d'eau salée dans un vaste bassin anti-salure.

Dans la mesure où les solutions (b) et (c) n'étaient pas significativement différentes du point de vue du coût des ouvrages et où la solution (c) apparaissait nettement préférable, d'une part sur le plan du rendement en perte d'eau douce et de l'introduction de sel dans le canal, d'autre part sur le plan de la simplicité de l'exploitation, elle seule a été retenue pour une étude approfondie sur modèle réduit.

\section{Description de l'écluse de Mardyck (Fig. 1)}

L'écluse de Mardyck a été spécialement aménagée, après des études approfondies effectuées par Sogreah à Grenoble et brièvement décrites au paragraphe 5 cidessous, pour remplir à la fois la fonction de sassement et la fonction de séparation de l'eau salée de l'eau douce selon la solution (c) inđiquée au paragraphe précédent.

Comme toutes les écluses de la liaison DunkerqueValenciennes, cet ouvrage se compose d'un sas de $144,60 \mathrm{~m} \times 12 \mathrm{~m}$, divisible en un moyen sas et un petit sas par une porte intermédiaire.

Ses bajoyers, son radier et ses têtes sont en béton armé.

Les portes extrêmes sont des portes levantes car, si le niveau du port est en général plus haut que celui du canal, il peut se produire dans certains cas une inversion de niveau. La porte intermédiaire est toutefois une porte busquée moins onéreuse et moins gênante du point de vue de la visibilité. L'usage de cette porte est donc interdit dans les rares cas où l'inversion de niveau signalée ci-dessus se produit.

L'originalité de l'ouvrage réside dans le fait que son sas peut recevoir et restituer à la fois de l'eau douce ou de l'eau salée par l'intermédiaire de deux systèmes d'aqueducs séparés :

- l'un, affecté à l'eau douce, gravitaire et débouchant dans le sas par des conduits latéraux;

- l'autre, affecté à l'eau salée, gravitaire ou commandé par des pompes et débouchant dans le sas par des perforations pratiquées dans le radier.

Ces aqueducs permettent d'échanger complètement l'eau dans le sas, en présence ou non d'un bateau, par substitution directe, en profitant du fait que, l'eau salée étant plus lourde, on peut conserver entre cette dernière et l'eau douce une interface relativement nette. 


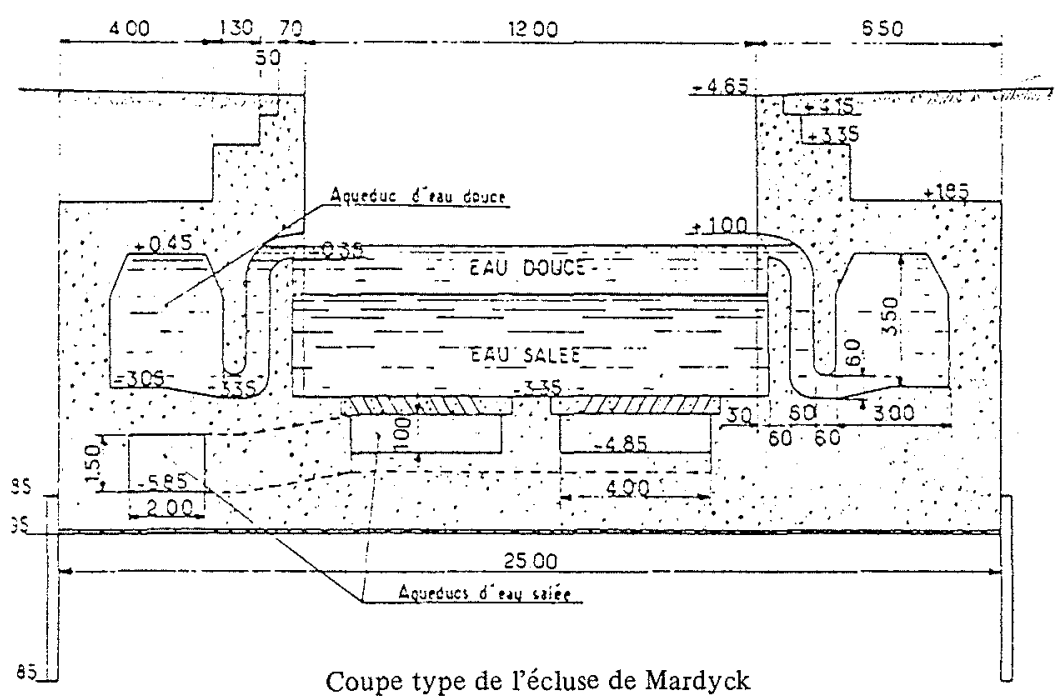

L'opération classique de mise à niveau dans l'écluse s'effectue toujours avec de l'eau salée. L'échange de l'eau salée par l'eau douce ou l'échange de l'eau douce par l'eau salée s'effectue toujours à niveau bas dans le sas.

Les pertes d'eau douce ont plusieurs causes :

a) $\mathrm{Au}$ niveau des siphons latéraux, l'aspiration de l'interface est de l'ordre de 0,25 à $0,30 \mathrm{~m}$ avec les conditions de débit adoptées. Il en résulte l'impossibilité pratique de récupérer toute l'eau douce du sas et la couche résiduelle s'étalera sur l'eau de mer lors de l'ouverture de la porte, sous l'effet de la différence des densités.

On peut réduire l'épaisseur de cette couche d'eau douce à condition de laisser pénétrer du sel dans les aqueducs latéraux. Le modèle réduit a mis en évidence un bilan global en sel meilleur malgré les diffusions induites dans les aqueducs.

b) $\mathrm{Au}$ niveau du radier, l'eau salée pénètre dans le sas sous forme de jets verticaux dont l'effet a été tudié sur un modèle réduit particulier. Bien que les vitesses de ces jets soient faibles, de l'ordre de $0,15 \mathrm{~m} / \mathrm{s}$, il se forme au début de l'injection et au-dessus de chaque orifice, de véritables champignons qui déferlent dès que leur hauteur atteint une dizaine de centimètres en entraînant une dispersion turbulente du sel dans l'eau douce.

c) Malgré l'échange d'eau, du sel est rejeté dans le canal soit parce qu'il sera resté au fond du sas une couche d'eau saumâtre en fin d'échange, soit par suite de fuites de vannes. Aussi une fosse anti-sel a-t-elle été prévue pour recueillir ces eaux saumâtres. Bien que profonde to $4 \mathrm{~m}$ au-dessous du niveau du canal, le passage des bateaux $y$ induit probablement une certaine diffusion par turbulence. Les eaux ainsi perdues sont reprises par deux pompes auxiliaires de $150 \mathrm{1} / \mathrm{s}$ chacune et rejetées dans l'eau de mer.

\section{Les essais sur modèle réduit}

\section{Description du modèle}

Le modèle réduit sur lequel a été étudiée et mise au point la solution retenue représentait le canal, sur une longueur de $320 \mathrm{~m}$, l'écluse et le bassin portuaire, sur une longueur de $290 \mathrm{~m}$, ces longueurs étant apparues nécessaires pour :

- réaliser les manoeuvres de convois ;

- obtenir des niveaux d'eau suffisamment constants de part et d'autre de l'écluse pendant les manoeuvres de convois ;

- étudier le déplacement éventuel de la langue salée au-delà du bassin anti-salure.

Pour toutes les dimensions du modèle (longueurs et hauteurs) a été choisie l'échelle $1 / 12,5$, ce qui a conduit à un modèle d'écluse plus grand que ceux habituellement réalisés, mais permettant, par ses dimensions, de mieux reproduire le détail des turbulences locales et des mélanges d'eau douce et d'eau salée dûs à ces turbulences.

Le mođèle était établi en similitude de Froude.

Le calcul avait mis en évidence que, dans le modèle, la perte de charge totale dans les aqueducs serait bien reproduite, mais que la perte causée par frottement aux parois serait plus grande qu'en réalité, ce qui n'est pas très gênant dans la mesure où son rôle n'apparaît pas très important.

Le pompage de l'eau salée a été réalisé sur modèle par une pompe à hélice horizontale, qui pouvait pomper dans les deux sens, comme envisagé dans la nature et dont la vitesse de rotation était réglée par un groupe Wait-Leonard. L'utilisation d'une vanne montée en série avec la pompe fournissait une autre possibilité de réglage du débit pendant les opérations d'éclusage.

Pour étudier le comportement de l'écluse, ont été mis en place des dispositifs permettant de mesurer :

- le niveau de l'eau dans les biefs et dans le sas;

- le débit de pompage et le volume total pompé ; - la salinité pendant et après éclusage (colorimétrie, densimétrie, conductimétrie). 


\section{Essais effectués}

Les essais effectués ont essentiellement concerné : - le réglage de la répartition du débit par les orifices de fond ;

- le réglage de la répartition des débits aspirés et refoulés par les aqueducs d'eau douce ;

- l'observation du comportement des différentes parties de l'ouvrage, sans convoi, avec convoi dans le sas, avec entrée et sortie d'un convoi, ce qui a conduit à préconiser deux modifications principales au projet initial de l'écluse :

- augmenter la largeur de l'écluse à sa partie inférieure pour réduire l'effet de pistonnage lors de l'entrée d'un convoi dans le sas et la vitesse d'écoulement entre le bajoyer de l'écluse et le convoi lors de la vidange ou du remplissage,

- prévoir la communication entre le sas et les aqueducs d'eau douce de telle sorte que l'eau salée introduite dans ces aqueducs demeure au fond de ceux-ci et éventuellement puisse être évacuée en priorité sans provoquer trop de mélange,

- observer l'efficacité d'ensemble des dispositifs, en évaluant le poids total de sel introduit dans le bassin anti-salure par cycle d'éclusage et les pertes d'eau douce qui en résultent.

\section{Conclusion des essais}

Les essais effectués ont conduit à quatre groupes de conclusions :

\section{a) Modifications du projet}

Différents ajustements ont été apportés à l'installation et ses conditions d'exploitation afin de réduire les pertes d'eau douce par échappement des aqueducs, pompage direct, mélange dans le sas, mélange sous le radier dans les chambres, mélange dans les aqueducs d'eau douce, recyclage de l'eau mélangée du bassin anti-salure, mélange dans le bassin anti-salure.

\section{b) Choix des caractéristiques principales des pompes}

Il a été préconisé des pompes réversibles à vitesse variable d'un débit total n'excédant pas $20 \mathrm{~m}^{3} / \mathrm{s}$ pour la hauteur manométrique totale de $3,70 \mathrm{~m}$ correspondant à une perte de charge de $1,50 \mathrm{~m}$ dans les aqueducs et à une chute de $2,20 \mathrm{~m}$ en phase de changement d'eau à la vidange.

\section{c) Spécialisations et spécifications pour les vannes}

Les essais ont conduit àdéfinir avec précision les caractéristiques principales des différentes vannes, leurs différents rôles et leurs conditions de manœuvre.

\section{d) Disposition pour les manouvres automatiques}

En raison de sa complexité, le fonctionnement de l'écluse ne pouvait être qu'automatique. La fin de la phase d'échange présentait une certaine difficulté de repérage parce que, d'une part l'interface de séparation des deux couches d'eau offre une épaisseur non négligeable, et d'autre part les détections de salinité par conductibilité électrique nécessitent de fréquents nettoyages et réétalonnages peu pratiques en exploitation. La solution retenue est basée sur le réglage de la combinaison volume/temps ou, ce qui revient au même, débit-temps. Elle entraîne en contre-partie un étallonnage précis des pertes de charge dans les circuits hydrauliques et la connaissance du volume déplacé par les bateaux en transit, volume qui intervient en déduction du volume d'échange à réaliser.

Trois paramètres gouvernent donc le système :

- la différence de niveau port-canal ;

- la durée de la phase d'échange ;

- le volume d'eau à échanger.

Le premier paramètre est facile à détecter. Il est affiché directement sur le pupitre de commande de l'écluse.

La durée d'échange a été fixée lors des études initiales à deux valeurs possibles, soit $10 \mathrm{mn}$ en hiver et $12 \mathrm{mn}$ en été, période d'étiage des eaux d'alimentation des canaux.

Le volume d'échange diffère entre hiver et été. Il est limité au strict volume d'eau contenu dans le sas, soit $8400 \mathrm{~m}^{3}$ en hiver. En été, pour obtenir l'économie maximale, on accroît ce volume à $10600 \mathrm{~m}^{3}$, ce qui introduit dans les aqueducs, en accord avec les résutats du modèle réduit,une quantité théorique d'eau salée égale à $50 \%$ de leur volume.

Grâce à un programme de calcul sur ordinateur, des tables à l'usage de l'éclusier ont été établies, une table en usage pour l'été l'autre pour l'hiver. Elles donnent, en fonction du volume des bateaux et de la dénivellation, le degré d'ouverture des vannes (échange de l'eau douce par l'eau salée) ou la vitesse des pompes (échange de l'eau salée par l'eau douce). Les pompes sont entraînées par l'intermédiaire d'un coupleur hydraulique.

\section{Constatations et mises au point effectuées a l'entrée en service de l'écluse}

\section{Etalonnage des circuits hydrauliques}

Les premiers essais réalisés sur l'écluse furent l'étalonnage des circuits hydrauliques. Effectués avant l'achèvement des travaux de raccordement avec les bassins du port, on disposait en amont de l'écluse, d'une portion de canal fonctionnant en réservoir et remplie d'eau douce. Il était facile de faire varier le niveau amont, d'établir un régime permanent et de mesurer les débits avec une batterie de moulinets placée dans l'extrémité du sas côté aval. Des lissages par moindres carrés permirent de donner des expressions analytiques des lois de pertes de charge. En appelant $Q$ le débit, en $\mathrm{m}^{3} / \mathrm{s}, N$ louverture des vannes, avec $0<N<1$, et $H$ la dénivellation mer-sas ou sas-canal selon le cas, en mètres, les résultats suivants ont été obtenus :

\section{a) Circuits gravitaires d'eau salée}

avec

$$
\begin{gathered}
Q=a H b \\
a=23,92 N^{0,82} \\
b=0,57 N^{-0,23}
\end{gathered}
$$


b) Circuits de pompage d'eau salée (les pompes ellesmêmes ayant été contrôlées sur barre par le constructeur)

$$
Q=9,13 H^{0,73}
$$

c) Circuits d'eau douce (deux aqueducs)

- sens écluse-canal

$$
\begin{aligned}
H & =2,8810^{-5} Q^{2,68} \\
\text {-sens canal-écluse } & =0,27110^{-3} Q^{2}
\end{aligned}
$$

\section{Mesures de salinité}

Deux campagnes de mesures de salinité ont été effectuées à Mardyck, la première avant l'ouverture de l'écluse au trafic, la seconde lorsque celui-ci est devenu suffisant, notamment en ce qui concerne les convois poussés de $3000 \mathrm{t}$.

Ces mesures ont été faites avec les détecteurs de salinité basés sur la conductibilité de l'eau. Toutefois, la nécessité de ne pas éloigner la sonde des appareils de mesure entraînait l'emploi d'un circuit de puisage de l'eau et d'amenée au capteur, circuit constitué d'une petite pompe électrique et de tuyaux plastiques.

L'appareillage était d'une excellente sensibilité, mais le principe de mesures introduisait une certaine imprécision, car, d'une part on n'obtenait pas une mesure ponctuelle mais une moyenne autour du point de prélèvement de l'eau, d'autre part les valeurs fournies par les appareils tenaient compte de tous les sels dissous et non du seul chlorure de sodium.

Le but des essais était essentiellement de confirmer les consommations en eau douce de l'écluse, de préciser les phénomènes d'échange dans les aqueducs latéraux et l'action des manœuvres des bateaux entrant dans le sas ou en sortant, car le modèle réduit avait montré un effet de piston important et des contre-courants dans les aqueducs latéraux et les circuits situés sous le radier.

Un grand nombre de paramètres interviennent dans le fonctionnement de l'écluse et il est difficile, avec un nombre limité de mesures, d'isoler le rôle de chacun et de trouver les lois de variation. De plus, les phénomènes de densité mettent en jeu des forces et des frottements très faibles, et l'on se heurte toujours à une certaine dispersion aléatoire des résultats. Aussi, après quelques recherches, ont été utilisées des méthodes statistiques et dégagées les tendances grâce à des calculs de corrélation.

\section{Essais hors navigation}

Les profils de salinité relevés dans le sas sont très similaires à celui observé sur le modèle réduit et la fígure 2.a donne leur allure générale.

A niveau bas, après échange de l'eau salée par l'eau douce, le profil relevé est tantôt strictement vertical, ce qui indique un sas parfaitement rempli d'eau douce, tantôt légèrement incurvé au niveau du radier. A niveau haut lors de l'échange inverse, la concentration en sel proche de celle de l'eau douce croît linéairement avec la profondeur sur le tiers environ de la hauteur d'eau, puis devient constante et égale à la concentration de l'eau de mer. Il n'y a donc pas d'interface au sens géométrique du terme, mais seulement une zone de transition.

A l'ouverture des portes de l'écluse :

- l'eau superficielle mais dense s'étale vers le port ; - la concentration dans le sas s'accroît lentement ; - la salinité crồt linéairement avec la profondeur dans les aqueducs latéraux.

Les valeurs moyennes obtenues sur une trentaine d'essais sont les suivantes :

- cote de l'eau dans le canal

- cote de l'eau dans le port

$0,70 \mathrm{NGF}$ ou

4,05 marine

$1,88 \mathrm{NGF}$ ou

5,23 marine

- concentration en sel de l'eau de mer

- concentration en sel de l'eau douce

- concentration moyenne dans le sas à niveau haut

- concentration moyenne dans les aqueducs

- débit moyen d'échange

$28,28 \mathrm{~g} / \mathrm{kg}$

$2,16 \mathrm{~g} / \mathrm{kg}$

$21,75 \mathrm{~g} / \mathrm{kg}$

$13,04 \mathrm{~g} / \mathrm{kg}$

$14,2 \mathrm{~m}^{3} / \mathrm{s}$

A niveau haut, après remplissage en eau salée, ces valeurs donnent les volumes équivalents ci-dessous : - volume moyen d'eau douce - volume moyen d'eau salée $2490 \mathrm{~m}^{3}$ $7430 \mathrm{~m}^{3}$ soit un rendement de l'échange d'eau de l'ordre de $75 \%$.

Dans les aqueducs latéraux, la concentration relevée correspond à un mélange de $1660 \mathrm{~m}^{3}$ d'eau douce avec $2440 \mathrm{~m}^{3}$ d'eau salée.

L'expérience montre que l'on a bien laissé pénétrer dans les aqueducs la quantité de sel théoriquement prévue mais qu'il ne s'y établit pas de stratification véritable, si bien qu'une partie du sel est même rejetée côté canal.

Dans une écluse comme celle de Mardyck, en l'absence de bateau, les volumes d'eau extraits du sas par pompage sont strictement égaux aux volumes qui y sont introduits. La masse d'eau qui sert d'interface entre l'eau douce et l'eau salée est donc déplacée alternativement dans les deux sens et l'on conçoit ainsi que l'interface soit plus diffus que dans un milieu au repos.

\section{Essais avec navigation}

Alors que l'on pouvait escompter un fort brassage de l'eau du sas et une destruction de la stratification, les essais ont révélé le contraire. Quand un bateau pénètre dans le sas en provenance du port, l'eau déplacée s'écoule sous le bateau, si bien que l'eau salée est rejetée et que l'interface s'abaisse. Ce phénomène est évidemment dû à la lenteur des manoeuvres du bateau et au fait que les hélices situées à l'arrière n'interviennent pratiquement pas dans le sas. Ainsi sur douze essais, tous effectués avec des convois de $3000 \mathrm{t}$ de charge utile et déplaçant $4000 \mathrm{~m}^{3}$, l'eau chassée du sas représente $1500 \mathrm{~m}^{3}$ d'eau douce et $2500 \mathrm{~m}^{3}$ d'eau salée et l'interface théorique moyen s'est abaissé de $1,21 \mathrm{~m}$ à $1,61 \mathrm{~m}$ sous la surface.

Lors de la sortie d'un convoi du sas vers le canal Dunkerque-Valenciennes, on est au contraire surpris 
a) Conceniration en sei sans novigction - Solt concentrotion without ship
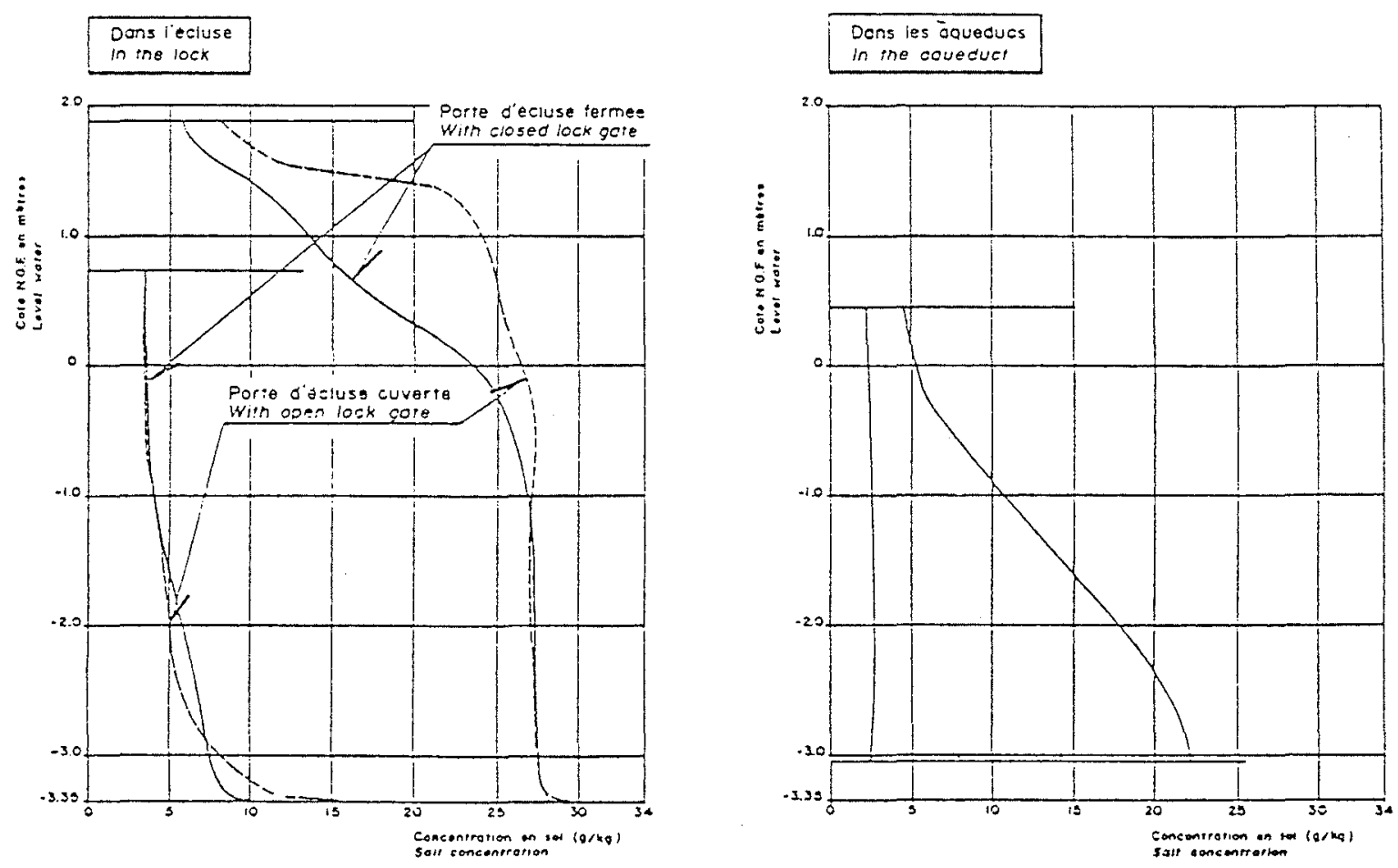

b) Concentrction en sel cvec novigation - Solt concentration with ship
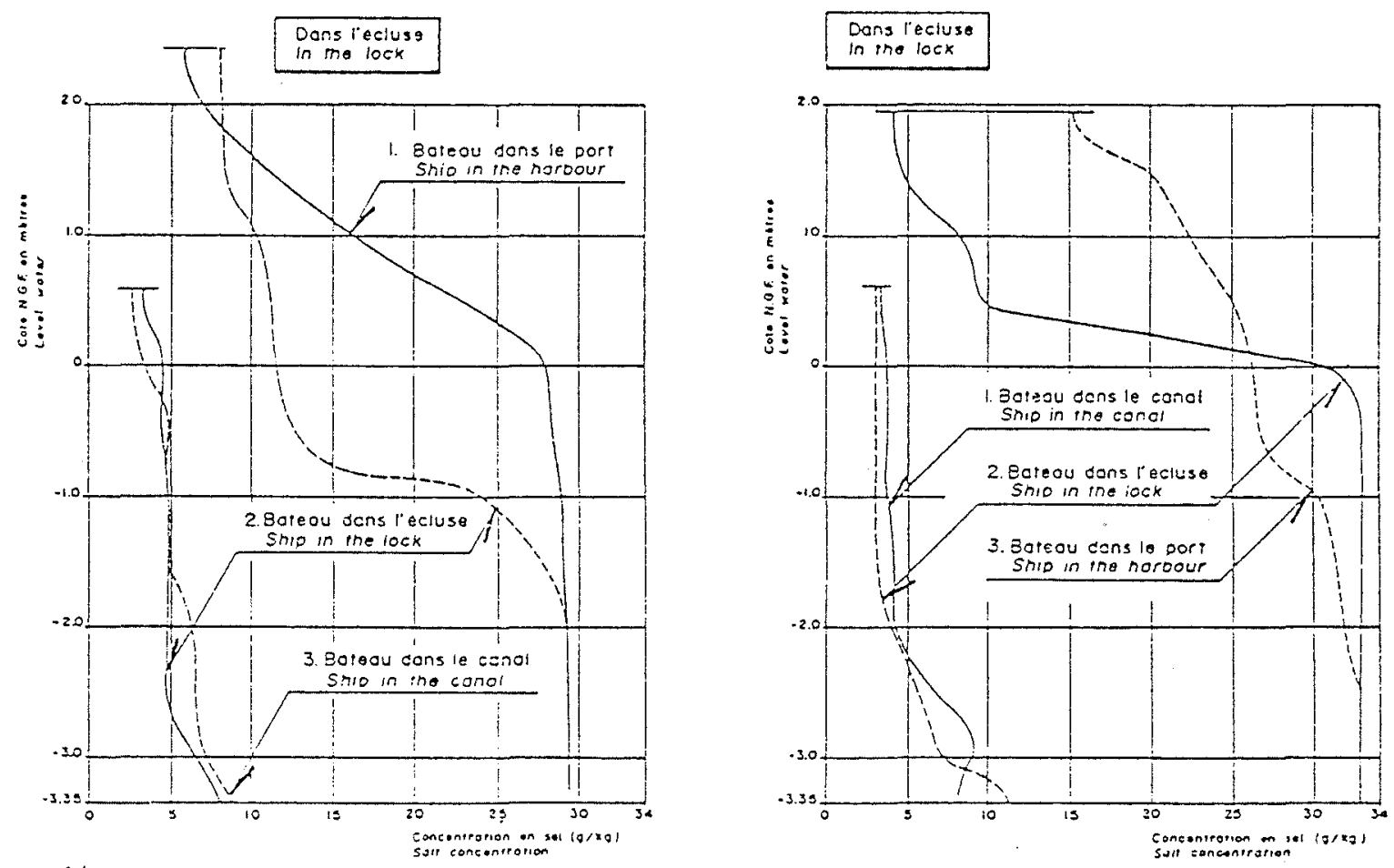

Figure 2 
de voir la salinité au fond du sas s'accroître d'une quantité équivalente à un apport de $250 \mathrm{~m}^{\mathrm{J}}$ d'eau de mer. Comme il est hautement improbable qu'une telle quantité provienne des seuls circuits situés sous le radier dont le volume ne représente que $750 \mathrm{~m}^{3}$ d'eau, il faut bien admettre qu'une partie du sel est réaspirée dans la zone anti-sel.

Le calcul confirme la vraisemblance de cette hypothèse. Les durées de manoeuvre des bateaux conduisent à un débit de remplissage du sas de l'ordre de $17 \mathrm{~m}^{3} / \mathrm{s}$. L'interface dans le bassin anti-sel est en moyenne à la côte $-4,70 \mathrm{NGF}$ et la côte limite de l'aspiration d'une saumure à $10 \mathrm{~g} / \mathrm{kg}$ peut atteindre $-6,00 \mathrm{NGF}$.

La surlargeur donnée au sas au-dessus des aqueducs latéraux avait pour objet de s'opposer aux contre-courants induits dans les aqueducs par le déplacement des bateaux. L'absence de toute variation de salinité dans le sas pendant les manoeuvres démontre l'efficacité de cette solution.

\section{Bilan général de consommation en eau douce}

Pour confirmer la valeur de la consommation en eau douce sur une longue période d'exploitation, les services du Port Autonome de Dunkerque ont assuré le pointage de toutes les manoeuvres et des relevés très fréquents de la salinité dans le bassin anti-sel pendant près de deux mois. L'exploitation de ces données sur ordinateur a confirmé que la consommation moyenne par cycle complet de l'écluse s'établissait bien à $2200 \mathrm{~m}^{3}$ perdus dans le sas et $1800 \mathrm{~m}^{3}$ par rejet et diffusion dans le bassin anti-sel.

\section{Essai d'interprétation théorique des résultats}

Ces essais ont été exposés par M. Quetin dans la conférence qu'il a prononcée en 1972 devant le Comité Technique de la Société Hydrotechnique de France.

Je n'y reviendrai pas, me bornant à ses propositions de définition d'un rendement global de l'écluse.

- Ce rendement peut être estimé égal au rapport entre le volume d'eau douce consommé et le volume total d'eau échangé. Ce rendement est à Mardyck d'environ $60 \%$. Il n'est pas exempt de critique, compte tenu des diffusions et brassages dans le bassin anti-salure.

- Une autre définition est possible, consistant dans le rapport du volume d'eau douce perdu dans le sas au volume d'eau contenu dans le sas pendant.l'échange. Le rendement est à Mardyck d'environ $75 \%$.

\section{Conclusion}

L'expérience de l'exploitation de l'ouvrage a permis de dégager plusieurs points .

- sur le plan de fonctionnement intrinsèque de l'ouvrage, la principale observation concerne le comportement du système à la fin de la substitution de l'eau salée à l'eau douce, juste avant le remplissage. L'observation de la salinité du bassin anti-salure montre une élévation rapide de cette dernière après 8 ou $9 \mathrm{mn}$ d'échange. On a d'ailleurs observé une certaine dérive dans l'apparition de ce seuil, liée sans doute à l'envasement des chambres de tranquillisation situées sous le sas.

En exploitation courante, on a ainsi été amené à faire fonctionner la pompe de $175 \mathrm{l} / \mathrm{s}$ pendant environ $20 \mathrm{~h} /$ jour, voire même en permanence, ce qui a pratiquement conduit à supprimer le fonctionnement en régime d'été, et même à n'échanger l'eau que pendant $8 \mathrm{mn} 30 \mathrm{~s}$. La perte d'eau douce au sassement est largement compensée par la réduction du pompage antisalure.

- Une autre mise au point a été effectuée lors de l'exploitation. On rouvre les vannes d'eau douce au moment des mouvements des convois vers le canal et vice-versa. Cette ouverture diminue la vitesse de l'eau au ras des orifices du fond et l'effort à foumir par les hélices pour mettre le bateau en mouvement. On réduit ainsi la diffusion de l'eau salée contenue dans les chambres de tranquillisation du fond du sas. Il serait ainsi sans doute souhaitable d'élargir un peu le sas de la future écluse.

- On a enfin observé que, pendant les périodes, en principe assez rares, où le canal est au-dessous de son niveau normal, le débit des siphons au remplissage du sas est insuffisant. Il se produit une dénivelée trop forte et l'eau douce est introduite dans le sas avec chute, d'où un mélange avec l'eau salée, du moins au début de l'échange. Il est alors nécessaire de réduire le débit de pompage pour l'adapter au débit de l'eau douce et de réduire la durée de l'échange.

En définitive, après 13 ans d'exploitation, le fonctionnement de l'écluse de Mardyck apparaît pleinement satisfaisante, assurant une séparation convenable de l'eau douce et de l'eau de mer. La mesure régulière de la salinité le long du canal en amont de l'écluse permet d'ailleurs de juger de l'efficacité du dispositif. 